\title{
riccafd
}

Revista Iberoamericana de Ciencias de la Actividad Física y el Deporte

\section{LA SOCIALIZACIÓN DE LAS PERSONAS MAYORES EN EL PARQUE BIOSALUDABLE}

\section{SOCIALIZATION OF THE ELDERLY IN OUTDOOR HEALTH} CIRCUITS

Romero-Reche, A. ${ }^{1}$; Martos-Fernández, P. ${ }^{1}$ y Hita-Alonso, C. ${ }^{1}$

${ }^{1}$ Romero-Reche, A. Profesor sustituto interino, Departamento de Sociología, Universidad de Granada (España) romeroreche@ugr.es

${ }^{1}$ Martos-Fernández, P. Profesora titular, Departamento de Sociología, Universidad de Granada (España) pmartos@ugr.es

${ }^{1}$ Hita-Alonso, C. Profesora titular, Departamento de Sociología, Universidad de Granada (España) cehita@ugr.es

Código UNESCO: 6301 Sociología cultural

Clasificación Consejo de Europa: 16. Sociología del deporte

Recibido el 15 de junio de 2015

Aceptado el 21 de septiembre de 2015

Correspondencia:

Romero-Reche, A.: romeroreche@ugr.es

\section{RESUMEN}

En los parques biosaludables, originalmente concebidos para la población madura y anciana, se encuentran usuarios de diferentes edades y con distintas formas de entender la actividad física. El presente trabajo intenta examinar las relaciones de las personas mayores con el resto de usuarios para determinar en qué medida dichos parques pueden cumplir alguna función social más allá del fomento de hábitos saludables. Para ello se ha llevado a cabo una serie de observaciones, participantes y no participantes, en tres parques de la ciudad de Granada, donde se ha visto que, si bien existe una proporción minoritaria pero importante de usuarios jóvenes (aproximadamente un tercio del total), estos 
tienden a evitar una interacción que parte de los usuarios de mayor edad buscan expresamente.

\section{PALABRAS CLAVE}

Parques biosaludables, mayores, socialización, interacción social

\section{ABSTRACT}

The outdoor health circuit, originally conceived to be used by the mature and the elderly, is a meeting point for users of different ages and with different conceptions of physical activity. This paper attempts to examine the social relations of the elderly with the rest of users, in order to determine to what extent the health circuits might be fulfilling any other social function beyond encouraging healthy habits. To that end, a series of participant and nonparticipant observations have been carried in three circuits in Granada, where it has been observed that, while there is a significant minority of young users (approximately a third of the total number of users), these tend to avoid the interactions that some of the elderly users actively try to engage in.

\section{KEY WORDS}

Outdoor health circuits, old age, socialization, social interaction

\section{INTRODUCCIÓN}

La proliferación de parques biosaludables por las ciudades españolas responde a la voluntad de ofrecer a las personas mayores una opción sin coste económico y fácilmente accesible para realizar una actividad física regular de mantenimiento. Pero como tantas otras infraestructuras promovidas por los poderes públicos, los parques biosaludables contemplan usos que difieren de su propósito original desde el momento en que distintos sectores de la ciudadanía y grupos sociales más o menos identificables se apropian de ellos y 
los convierten en escenario de prácticas que se consolidan y se transforman con el paso del tiempo.

Más allá de casos específicos de «invasión» del parque por practicantes de deportes urbanos (skaters, parkour, etc), en este trabajo nos hemos interesado por la convivencia de los usuarios originales con otros grupos de edad que también se ejercitan en el mismo, comparando sus respectivos modelos de práctica deportiva y de interacción social, y observando las relaciones que se dan entre unos y otros.

\section{La resocialización de la tercera edad}

Es de sobra conocido que el proceso de socialización abarca toda la vida y que cada etapa vital cuenta con entornos e instituciones que guían este proceso en una determinada dirección. Históricamente, desde los poderes públicos se ha prestado más atención a la socialización en las etapas más tempranas en las biografías de los individuos (la familia y el sistema educativo son buenos ejemplos). Sin embargo el colectivo de mayores - a partir de los 60 años- ha visto mermada no solo la atención pública sino también la capacidad de crear entornos para socializarse al producirse paulatinamente alejamiento del trabajo, emancipación de los hijos, desaparición de amistades, falta de instituciones ad hoc, etc... Además, como señala Gil Calvo ${ }^{1}$, este colectivo ha sido visto como una carga social (por el coste económico que supone mantenerlo con cierta calidad de vida y por la atención de familiares que reclama). Dicho coste social y económico irá en aumento puesto que este colectivo es ya el más numeroso de la historia y se espera que no haga sino aumentar (incluso algún estudio reciente sobre patrones de fertilidad en la población de los países desarrollados, que sugiere que proporcionalmente dicho colectivo no tendrá el peso que se le auguraba hace unos años, observa que sigue planteando desafíos en términos de coste social y económico ${ }^{2}$ ).

No obstante, también este colectivo se sitúa en el punto de mira de las instituciones públicas por la cantera de votos que puede suponer y por el hecho 
de que puede mutar en un colectivo con una vida más o menos plena, activa, consumista e incluso laboralmente provechosa. El llamado «poder gris» ${ }^{1}$, puede convertirse en socialmente significativo y orientar los poderes públicos hacia sus propios intereses, sobre todo si tenemos en cuenta que los «futuros mayores», serán más cultos, más sanos y estarán más informados. Para todo ello se considera de vital importancia el mantenimiento de la actividad física y unas relaciones sociales continuadas y satisfactorias.

Puesto que «ser mayor» es una construcción social interiorizada por los individuos y puede crear estigmatización de la vejez ${ }^{3}$, nada indica que esa construcción social no pueda ser modificada, aun siendo conscientes del proceso inevitable e irreversible del envejecimiento y la muerte. En la modificación de esta imagen estereotipada y negativa es donde adquiere relevancia este interés doble, tanto del propio colectivo como de los poderes públicos, por incorporarse e incorporarlos a prácticas en principio de colectivos de menos edad, como la actividad física continuada y regular.

De esta necesidad han surgido los parques biosaludables, -lo que para algunos autores supone en gran medida una colonización de los espacios públicos ${ }^{4}$-, pensados para personas «menos jóvenes» y que pueden cumplir una doble función: socialización a través de la interacción con otros usuarios y la mejora física y mental de los mismos. Es decir, alterar o frenar en gran medida los rituales de paso a la tercera edad que señalan Puig y Mosquera ${ }^{3}$ : la jubilación que supone el abandono de una actividad cotidiana y rutinaria en torno a la cual se ha organizado la vida; la alteración del espacio-tiempo, dependiente en la etapa de la modernidad del empleo; el empobrecimiento económico; la soledad; el deterioro corporal y la muerte.

La actividad física de los mayores se está incrementando y no hay razones para suponer que no lo siga haciendo. El poder gris bien podría ser una realidad en unas cuantas décadas. 
Sociología de la actividad física de las personas mayores

Desde el punto de vista demográfico, el importante crecimiento de la población de más edad se está produciendo a la vez que mejoran ostensiblemente los niveles de salud.

Una vez que ha sido efectiva la conquista de años a la vida, el gran objetivo que se persigue ahora es el de añadir vida a los años, es decir, dotar a esos años de más riqueza personal y social para que el individuo pueda disfrutarlos en la mayor medida posible. Pues bien, en este contexto uno de los elementos que más puede contribuir a la mejora de calidad de vida es la participación en actividades diversas de ocio y tiempo libre, entre las cuales destacan aquellas relacionadas con el ámbito físico-deportivo ${ }^{5}$.

En efecto, el desarrollo de alguna actividad física es uno de los elementos que más contribuyen para alcanzar una vejez gratificante, no sólo en el terreno puramente físico sino también en el social ${ }^{6}$.

De acuerdo con la Encuesta de Hábitos deportivos de los españoles de $2010^{7}$, el $45 \%$ de las personas que se ejercitan regularmente lo hacen en espacios públicos, sea en ámbitos urbanos o rurales. En las sociedades urbanizadas contemporáneas, los hábitos saludables más recomendados por las autoridades sanitarias tienden a ser la alimentación equilibrada y el paseo, cuya práctica regular contrarrestaría las secuelas perniciosas de la vida sedentaria. $\mathrm{Y}$, en efecto, el paseo es la tercera actividad de tiempo libre que más mencionan los encuestados, continuando el patrón observado en las encuestas sobre hábitos deportivos de 2005 y 2000. El parque biosaludable ofrece un complemento de ejercicio físico a dicha actividad regular, fácilmente incorporable a la rutina deportiva de las personas, como han manifestado algunos de los entrevistados en nuestro estudio (existe incluso una «ruta del colesterol» oficiosa que establece el parque biosaludable como escala obligatoria en el paseo por prescripción facultativa). 
La encuesta de 2010 muestra además un importante incremento en la actividad físico-deportiva de las personas mayores, que alcanza un 19\% tras partir de niveles mínimos en 1980. Estas personas manifiestan dos razones fundamentales para practicar dichas actividades: la ejercitación (en un 74\%) y la salud (en un $75 \%)^{7}$. De nuevo, el parque biosaludable parece emerger como escenario privilegiado donde podrían cristalizar estas tendencias, en tanto infraestructura congruente con un discurso público que incentiva la actividad física, junto con toda una serie de transformaciones en las pautas de comportamiento y roles tradicionales, de un sector de la población de incuestionable relevancia cuantitativa y cualitativa, que podría comenzar a reclamar espacios sociales distintos de los que hasta ahora se les venían adjudicando.

\section{MÉTODO}

La investigación que se ha llevado a cabo tiene un carácter exploratorio, dadas la práctica inexistencia de estudios sobre los parques biosaludables desde una perspectiva sociológica y las limitaciones de los medios de que se disponía. Durante el mes de abril de 2014, en sesiones de mañana y tarde, y durante todos los días de la semana (de lunes a domingo, incluyendo también festivos), un equipo compuesto por 30 observadores ha combinado la observación participante y no participante en tres parques de la ciudad de Granada: Joaquina Eguaras (con un total de 216 observaciones realizadas), García Lorca (229 observaciones) y Bola de Oro (98 observaciones). Se eligieron dichos parques atendiendo a dos criterios: 1) ubicación geográfica: norte (Joaquina Eguaras), centro (García Lorca) y sur (Bola de Oro), y 2) perfil sociodemográfico y características del entorno: clase media-baja (Joaquina Eguaras), población con una media de edad mayor, clase media alta (García Lorca), clase media joven en una zona en expansión (Bola de Oro).

Los observadores se organizaron en parejas, compuestas cada una por un observador participante y un observador no participante; mientras éste se mantenía a cierta distancia de las instalaciones, tomando nota de las 
características «externas» de cada usuario en una ficha de observación, el participante se ejercitaba en los aparatos, escuchaba las conversaciones entre usuarios cuando estas se producían, y en ocasiones interactuaba directamente con ellos, registrando igualmente sus observaciones en las fichas correspondientes.

\section{RESULTADOS}

En cuanto a la representación de grupos de edad entre los usuarios de los parques, la observación ha arrojado los siguientes porcentajes: un 3’31\% de menores de 13 años, un 2'21\% entre 13 y 17 años, un 16'39\% de 18 a 25 años, un 17 '13\% entre 26 y 39 años, un 38'86\% entre 40 y 64 años, y un 22 '1\% de 65 o más.

Como queda patente, el grupo mayoritario no lo componen los usuarios de la tercera edad, sino los de mediana edad, que constituyen casi dos quintas partes de la muestra. Si lo unimos al grupo de la tercera edad, que en efecto representa algo más de una quinta parte, los usuarios con 40 años o más alcanzan en torno al $60 \%$, es decir, tres quintas partes del total. Fuera de dicho bloque quedan, por un lado, el conjunto relativamente insignificante de los menores de edad (dividido en dos categorías que suman un escaso $5 \%$ del total, lo cual es congruente con el uso anecdótico del parque biosaludable que cabe esperar de niños y adolescentes, pues cuentan en el entorno inmediato con otras posibilidades de ocio más afines a sus intereses), y otro bloque más voluminoso, el de los adultos «jóvenes» (que comprende a los jóvenes «propiamente dichos», hasta los 25 años, y a los «nuevos jóvenes» de entre 26 y 39).

Estos datos dibujan lo que podríamos llamar «relación cuantitativa de fuerzas» en el espacio del parque biosaludable: una mayoría de personas maduras y ancianas que hace uso de las instalaciones, de acuerdo con su función manifiesta, para contrarrestar los efectos de una vida sedentaria que empiezan a hacerse más presentes a partir de dichas etapas, frente a una asistencia 
menor pero significativa de personas jóvenes que integran el parque biosaludable dentro de su rutina de ejercicio físico cotidiano.

Cuando observamos qué proporción de los usuarios de cada grupo de edad llegan solos o acompañados al parque biosaludable (Tabla 1), vemos que, si bien la opción de ir solos supera generosamente la mitad de cada grupo de edad adulto (no así en el caso de los menores de edad, que van acompañados casi siempre), son los mayores los más propensos a hacerlo, en un 71 '7\%.

Tabla 1. Solo/ Acompañado según la variable edad. Fuente: Martos Fernández, Hita Alonso \& Romero Reche ${ }^{8}$.

\begin{tabular}{lcccccc}
\hline Llega & $\begin{array}{c}\mathbf{0 - 1 2} \\
\text { años }\end{array}$ & $\mathbf{1 3 - 1 7}$ & $\mathbf{1 8 - 2 5}$ & $\mathbf{2 6 - 3 9}$ & $\mathbf{4 0 - 6 4}$ & $\begin{array}{c}\mathbf{6 5} \mathbf{0} \\
\text { más }\end{array}$ \\
\hline Solo/a & 1 & 2 & 57 & 58 & 144 & 86 \\
& $\left(5^{\prime} 6 \%\right)$ & $\left(16^{\prime} 7 \%\right)$ & $(64 \%)$ & $\left(62^{\prime} 4 \%\right)$ & $\left(68^{\prime} 2 \%\right)$ & $\left(71^{\prime} 7 \%\right)$ \\
Acompañado/a & 17 & 10 & 32 & 35 & 67 & 34 \\
& $\left(94^{\prime} 4 \%\right)$ & $\left(83^{\prime} 3 \%\right)$ & $(36 \%)$ & $\left(37^{\prime} 6 \%\right)$ & $\left(31^{\prime} 8 \%\right)$ & $\left(28{ }^{\prime} 3 \%\right)$ \\
Total & 18 & 12 & 89 & 93 & 211 & 120 \\
& $(100 \%)$ & $(100 \%)$ & $(100 \%)$ & $(100 \%)$ & $(100 \%)$ & $(100 \%)$ \\
\hline
\end{tabular}

Sin embargo, es ese mismo grupo de edad el que más tiende a interactuar con otros usuarios (Tabla 2): un 35'8\% de los usuarios de 65 años o más entablaron, o intentaron entablar, algún tipo de contacto o relación con otras personas en el parque biosaludable. En términos porcentuales, es del doble que en el grupo de edad inmediatamente anterior (18'5\%). Obviando a los menores de edad que, como hemos visto, acuden acompañados, el grupo menos dispuesto a relacionarse con otros usuarios es el de los que tienen de 26 a 39 años.

Tabla 2. Interacción según la variable edad. Fuente: Martos Fernández, Hita Alonso \& Romero Reche 8 .

\begin{tabular}{lcccccc}
\hline $\begin{array}{l}\text { Interactúa con } \\
\text { otros usuarios }\end{array}$ & $\begin{array}{c}\mathbf{0 - 1 2} \\
\text { años }\end{array}$ & $\mathbf{1 3 - 1 7}$ & $\mathbf{1 8 - 2 5}$ & $\mathbf{2 6 - 3 9}$ & $\mathbf{4 0 - 6 4}$ & $\mathbf{6 5}$ o más \\
\hline Sí & 0 & 1 & 15 & 8 & 39 & 43 \\
& $(0 \%)$ & $\left(8^{\prime} 3 \%\right)$ & $\left(16^{\prime} 8 \%\right)$ & $\left(8^{\prime} 6 \%\right)$ & $\left(18^{\prime} 5 \%\right)$ & $\left(35^{\prime} 8 \%\right)$ \\
No & 18 & 11 & 74 & 85 & 172 & 77 \\
& $(100 \%)$ & $\left(91^{\prime} 7 \%\right)$ & $\left(83^{\prime} 1 \%\right)$ & $\left(91^{\prime} 4 \%\right)$ & $\left(81^{\prime} 5 \%\right)$ & $\left(64^{\prime} 2 \%\right)$ \\
Total & 18 & 12 & 89 & 93 & 211 & 120 \\
& $(100 \%)$ & $(100 \%)$ & $(100 \%)$ & $(100 \%)$ & $(100 \%)$ & $(100 \%)$ \\
\hline
\end{tabular}




\section{DISCUSIÓN}

Observaciones sobre el entorno

Como ya se ha dicho, cada parque biosaludable se inserta en un contexto geográfico y social característico, respecto al cual se han recogido tanto los comentarios de los usuarios como las aportaciones de los observadores. En el caso del parque del norte, Joaquina Eguaras, son frecuentes las observaciones sobre incivismo (presencia de perros sueltos, heces que no se recogen) y las advertencias sobre el peligro que representan los ladrones; esta última es la excusa con la que algunas personas del grupo de la tercera edad entablan la interacción, por iniciativa propia, con nuestros observadores en dicho parque.

En el caso de Bola de Oro, el «incivismo» tiene otro perfil: el de los grupos de adolescentes y jóvenes que aprovechan el relativo aislamiento del parque con respecto al entorno urbano para reunirse a beber cerveza y dejan las botellas vacías en el suelo. Por otra parte, los aparatos del parque biosaludable no propician la interacción entre usuarios: son individuales en su inmensa mayoría (es decir, no están dispuestos por parejas, como ocurre en otros parques) y se encuentran alejados entre sí. Por ejemplo, un usuario de ochenta años, vestido con ropa deportiva (lo cual es significativamente menos frecuente en los usuarios de mayor edad que en los jóvenes ${ }^{8}$ ), protestaba por las botellas tiradas en el suelo, pero al mismo tiempo sostenía que era el parque más completo y el que gozaba de mejor mantenimiento.

Pautas de «llegada»

El acceso de los usuarios al parque biosaludable también proporciona algunas pistas tanto sobre el lugar que ocupa en su rutina diaria, si es el caso, como sobre sus objetivos al hacer uso del mismo.

Observamos tres modelos básicos: 1) como escala dentro de una ruta de ejercicio físico (sea ciclismo, jogging, marcha, o sencillamente una caminata 
por prescripción facultativa, como en el caso de la usuaria de 70 años del parque Bola de Oro que decía recorrer con frecuencia la llamada «ruta del colesterol"); 2) como destino principal (es decir, como actividad físico-deportiva prioritaria, y no como complemento a otras), y 3) como "capricho del momento", cuando se usan los aparatos sencillamente porque están disponibles, en el curso de un paseo.

\section{Pautas de actividad física}

A este respecto, identificamos tres factores que se pueden combinar entre sí: 1) duración de la actividad, 2) intensidad, y 3) «corrección» de la misma (es decir, uso acorde con las instrucciones de los aparatos y con las condiciones físicas del usuario). El uso correcto es poco común en todos los grupos de edad (e inexistente en el caso de los menores, que emplean los aparatos para jugar) de la muestra observada. Por otra parte, si entendemos el uso de ropa deportiva como indicador de intencionalidad de la visita al parque o de compromiso con la actividad física, podemos llegar a conclusiones erróneas en el caso de los usuarios de mayor edad, mucho menos dados a vestir ropa deportiva tanto si acuden expresamente a ejercitarse en el parque (según lo manifiestan a nuestros observadores) como si lo hacen por inspiración del momento. El uso de otros accesorios deportivos (como, por ejemplo, pesas), son claramente indicativos de intencionalidad y compromiso, pero no se han observado en los grupos (si bien algunos de los usuarios entrevistados afirmaron hacerlo en otras ocasiones).

\section{Pautas de interacción}

Por un lado están los que acuden acompañados al parque. Entre ellos, distinguimos a los grupos familiares y a los amigos. En ambos tipos se dan casos en los que la interacción se mantiene en un tono más o menos lúdico (que generalmente lleva aparejado un uso igualmente «lúdico» de los aparatos, y una intensidad y duración menores del ejercicio), y otros en los que la interacción se suspende mientras dura la actividad física (ya se trate de amigos 
que se ejercitan juntos, o de adultos que aprovechan para hacerlo mientras los niños juegan). Es decir, la actividad física puede ser secundaria con respecto a la interacción (es una excusa para continuar interactuando), o la interacción puede estar subordinada a la actividad física (bien porque se suspenda temporalmente, bien porque esté al servicio de dicha actividad).

Los que acuden solos pueden interactuar o no con otros usuarios. En un extremo, se encuentran quienes se aíslan deliberadamente del entorno empleando auriculares y mostrando una actitud refractaria al contacto (menores de 40 años en todos los casos observados), y en el opuesto, los que buscan con la mirada a los demás usuarios y aprovechan cualquier ocasión para relacionarse (mayores de 40 años en la mayoría de casos observados; algunos de los usuarios han expresado dicha intención a nuestros observadores). Entre un extremo y otro, se da toda una gradación de posibilidades: así, quienes se contentan con (en palabras de uno de los usuarios) «ver al personal".

Los «detonantes» de la interacción son de diversos tipos: 1) Relacionados con la propia actividad física: indicaciones sobre el uso correcto de los aparatos, consejos para optimizar el ejercicio, comentarios sobre la mayor o menor dificultad del ejercicio. 2) Relacionados con el entorno físico, urbano o social: comentarios sobre las infraestructuras, sobre la zona de la ciudad, sobre el clima. 3) Normas de cortesía: la gestión del tiempo en los aparatos, cesiones de turno, saludos, agradecimientos y despedidas.

Los patrones observados denotan la coexistencia de distintas concepciones sobre la actividad deportiva, vinculadas con diferencias de carácter cultural, y con distintas demandas tanto en lo físico como en social y afectivo. Por un lado se encuentra la visión individualista del deporte, presente sobre todo en los menores de 40 años: la actividad física es una parcela importante de la vida cotidiana, que tiene su espacio reservado en la rutina diaria (que ha de configurarse en torno a las exigencias de la ocupación laboral), pero no debe desbordar sus límites ni cumplir cualquier otra función distinta de la puramente 
deportiva (nuestros observadores han aventurado el posible interés de algunos usuarios de este perfil hacia otros usuarios coetáneos del sexo opuesto; queda apuntado como especulación, pero en todo caso no se trataría del contacto intergeneracional que concierne a nuestro estudio). Por otro, se encuentra la concepción del deporte como «mal necesario", una actividad que se lleva a cabo por motivos de salud más o menos manifiestos, como excusa para salir a la calle en quienes se ven "forzados» al ocio tras jubilarse, incluso para sentirse participante de una actividad compartida con otros, pero en todo caso no como fin en sí mismo o no como fin principal.

\section{CONCLUSIONES}

Dentro de las ya mencionadas limitaciones del estudio exploratorio, en la muestra observada se aprecian las siguientes tendencias cuyo alcance sería conveniente investigar en estudios ulteriores:

1) Las personas mayores, destinatarios originales del parque biosaludable, son las que con más frecuencia acuden solas al mismo y las que con más frecuencia buscan el contacto con otros usuarios.

2) Los usuarios más jóvenes, «intrusos» en unas infraestructuras que no se han creado específicamente para ellos, son bienvenidos por los usuarios mayores como fuentes potenciales de una interacción social significativa.

3) La concepción de la actividad física por parte de estos usuarios jóvenes, que usan el parque únicamente para ejercitarse, les conduce a rechazar o desincentivar esa posible interacción social significativa con los usuarios mayores.

4) El parque biosaludable ofrece una posibilidad de contrarrestar en cierta medida el proceso de «expulsión» de la vida activa que supone la transición a la vejez. En la medida en que se mantiene la separación intergeneracional, dicha posibilidad sigue sin materializarse, y el parque se convierte en otro 
escenario donde representar, asumir e interiorizar el rol social de persona anciana.

\section{REFERENCIAS}

1. Gil Calvo E. El poder gris. Barcelona: Mondadori; 2003

2. Collins J, Richards OJ. Evolution, Fertility and the Ageing Population. Australia; 2014. [actualizado 4 enero 2015, citado 7 jun 2015]. Disponible en:

http://papers.ssrn.com/sol3/papers.cfm?abstract_id=2208886

3. Puig i Barata N, Mosquera González MJ. Género y edad en el deporte. En: García Ferrando M, Puig i Barata N, Lagardera Otero F, editores. Sociología del deporte. 3a ed. Madrid: Alianza; 2009. p. 99-126.

4. Sánchez Martín R, Capell Maymó M. Las lógicas del deporte en la calle: espacios, practicantes y socialidades en Barcelona. Apunts Educación física y deportes. 2008; 91: 44-53.

5. Latiesa M, Martos P, Puertas I. Actividad física, deporte y salud en la población granadina. Cáceres: I Congreso de la Asociación Española de Ciencias del Deporte; 2000. [citado 20 enero 2015]. Disponible en:

http://www.eweb.unex.es/eweb/cienciadeporte/congreso/00\%20cac/AF/7granada.pdf

6. Lehr UM. La calidad de vida en la tercera edad: una labor individual y social. En: Buendía J, comp. Envejecimiento y psicología de la salud. Madrid: Siglo XXI; 1994. p. 353-372.

7. García Ferrando M, Llopis Goig R. Ideal democrático y bienestar personal. Encuesta sobre los hábitos deportivos en España 2010. Madrid: Centro de Investigaciones Sociológicas y Consejo Superior de Deportes; 2011.

8. Martos Fernández $\mathrm{P}$, Hita Alonso $\mathrm{C}$, Romero Reche A. Biosaludando. Los parques biosaludables en Granada: interacción social y actividad físico-deportiva. En: Llopis Goig R, dir. Crisis, cambio social y deporte. Valencia: Nau Llibres; 2014.

\section{Referencias totales citadas:8}

Referencias citadas correspondientes a la Rev Ib CC Act Fis Dep: 0 